\title{
Editorial
}

\section{Recent Advances in Lunar Exploration Using Radar and Microwave Techniques}

\author{
Jing Li $\mathbb{D}^{1},{ }^{1}$ Zhiguo Meng $\mathbb{D}^{1},{ }^{1}$ and Alexander Gusev ${ }^{2}$ \\ ${ }^{1}$ College of GeoExploration Sicence and Technology, Jilin University, Changchun 130021, China \\ ${ }^{2}$ Kazan Federal University, Kazan, Russia \\ Correspondence should be addressed to Jing Li; inter.lijing@gmail.com
}

Received 17 June 2019; Accepted 17 June 2019; Published 7 July 2019

Copyright (C) 2019 Jing Li et al. This is an open access article distributed under the Creative Commons Attribution License, which permits unrestricted use, distribution, and reproduction in any medium, provided the original work is properly cited.

Since the successful launch of China's Chang'E (CE), Japan's SELENE, India's Chandrayaan, and America's LRO lunar orbiters, especially the Yutu rover onboard CE-3 mission, we have gained a further understanding about the Moon. Compared to the previous lunar missions, the microwave techniques have been paid more attention in recent mission, such as the lunar radar sounder (LRS) onboard SELENE, microwave sounder (CELMS) onboard CE-1/2, miniature synthetic aperture radar (Mini-SAR) onboard Chandrayaan, and Miniature Radio-Frequency (Mini-RF) instrument onboard LRO lunar orbiters, and the lunar penetrating radar (LPR) on board the Yutu rover in CE-3 mission. The microwave signal can penetrate the lunar regolith to some depth, and it is sensitive to the regolith thermophysical parameters. In the last five years, abundant exciting results have been revealed by the microwave data. However, the comparison among the findings using different data sources is critically absent. How to synthesize the microwave data, including the orbital and in situ measurements, to further understand the substrate thermophysical features of the Moon is one of the important research topics for the current lunar exploration. In this special issue, a few papers that address such issues are included.

11 papers were received before the deadline time. After strict review, 5 papers were accepted, and the other 6 were rejected. Prof. Li mainly sponsored 5 papers about the lunar penetration radar, Prof. Meng mainly sponsored 2 papers about the microwave observations of the Moon, and the others were processed by Prof. Gusev.

Of the 5 received papers, one addresses the structural analysis of lunar regolith from LPR CH-2 data based on adaptive $f$-x empirical mode decomposition (EMD). B. Hu et al. used the adaptive $\mathrm{f}-\mathrm{x}$ EMD based dip filter to extract the rock in the regolith indicating the degree of rock enrichment and highlighting regolith-basement interface. Their results locate the position of each rock and highlight the contact interface of regolith and the basement rock.

The paper "Disk-Integrated Lunar Brightness Temperatures Between 89 and $190 \mathrm{GHz}$ " presents the measurements of the disk-integrated brightness temperature of the Moon at $89,157,183$, and $190 \mathrm{GHz}$ which are presented for phase angles between $-80^{\circ}$ and $50^{\circ}$ relative to full Moon. M. J. Burgdorf et al. demonstrate the potential of weather satellites for finetuning models and establishing the Moon as an extremely accurate calibration reference.

In the paper "Study of the Chang'E-2 Microwave Radiometer Data in the Lunar Polar Region," F. Yang et al. used four-channel microwave radiometer (MRM) data with frequencies of $93 \mathrm{GHz}, 7.8 \mathrm{GHz}, 19.35 \mathrm{GHz}$, and 37 $\mathrm{GHz}$ to investigate the properties of lunar surface such as regolith thickness, dielectric constant, and titanium abundance within a depth of several meters in middle and low latitudes. The purpose of this work is to take a close look at MRM data in the polar regions of the Moon and analyze the characteristics of the brightness temperature (TB) in permanently shadowed regions (PSRs), especially where evidence of water ice has been found.

The paper "Several Geological Issues of Schrödinger Basin Exposed by CE-2 CELMS Data" evaluates the thermophysical features of Schrödinger basin using the Chang'E-2 microwave sounder (CELMS) data. Z. G. Meng et al. gave a new geological view according to the brightness temperature 
and emissivity maps and proved the applicability of the CELMS data applying in high latitude regions to a certain extent.

In the paper "Lunar Radiometric Measurement Based on Observing China Chang'E-3 Lander with VLBI-First Insight," Han et al. presented the current status and preliminary result of the OCEL, mainly focusing on the determination of the lander position. This accurate analysis is useful for optimizing OCEL sessions of the Chang'E-3 lunar lander.

\section{Conflicts of Interest}

All authors declare that there are no conflicts of interest regarding the publication of this article.

Jing $L i$

Zhiguo Meng

Alexander Gusev 

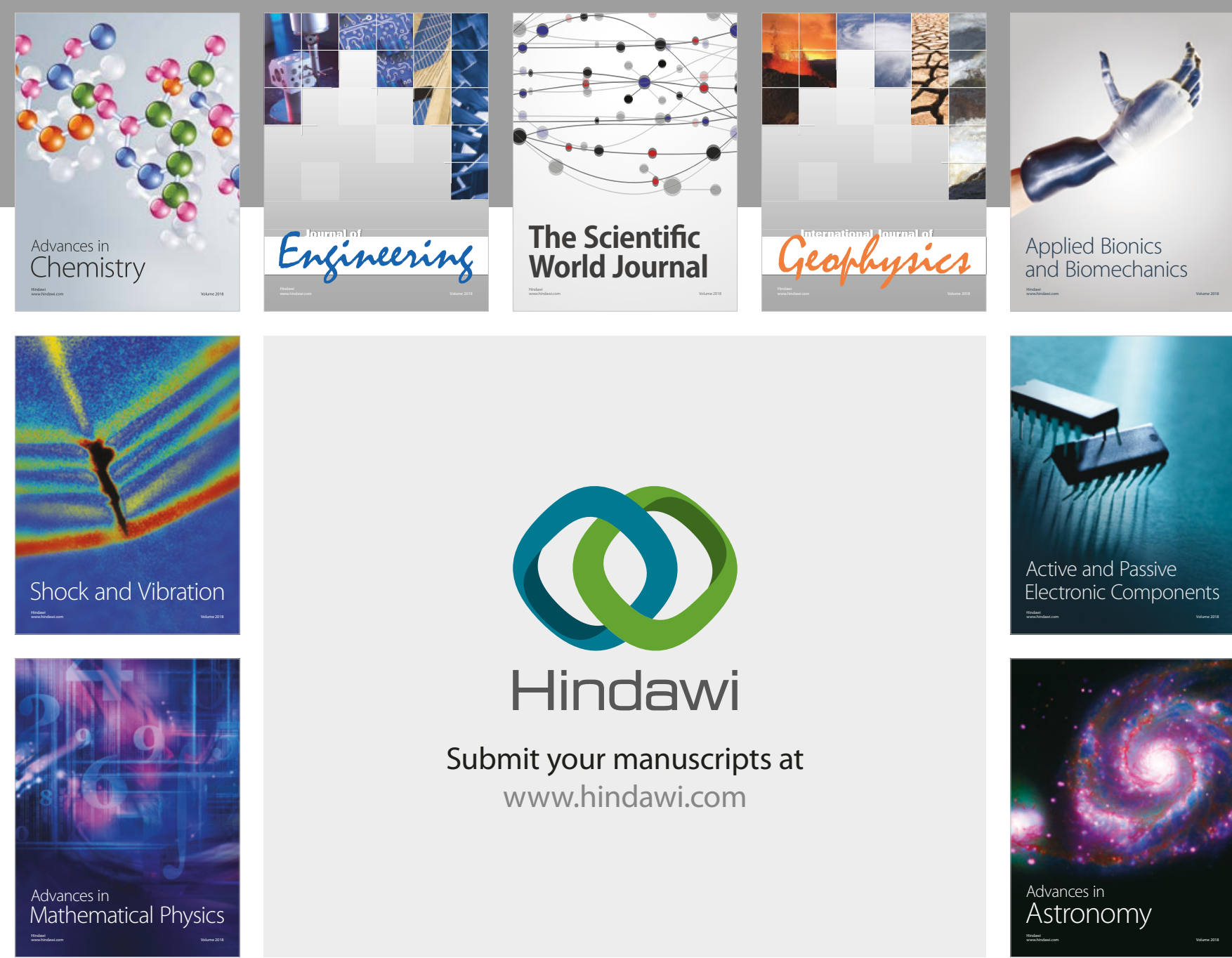

Submit your manuscripts at

www.hindawi.com

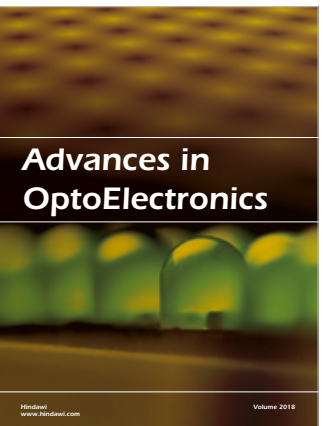

\section{Rotcting Machinery}
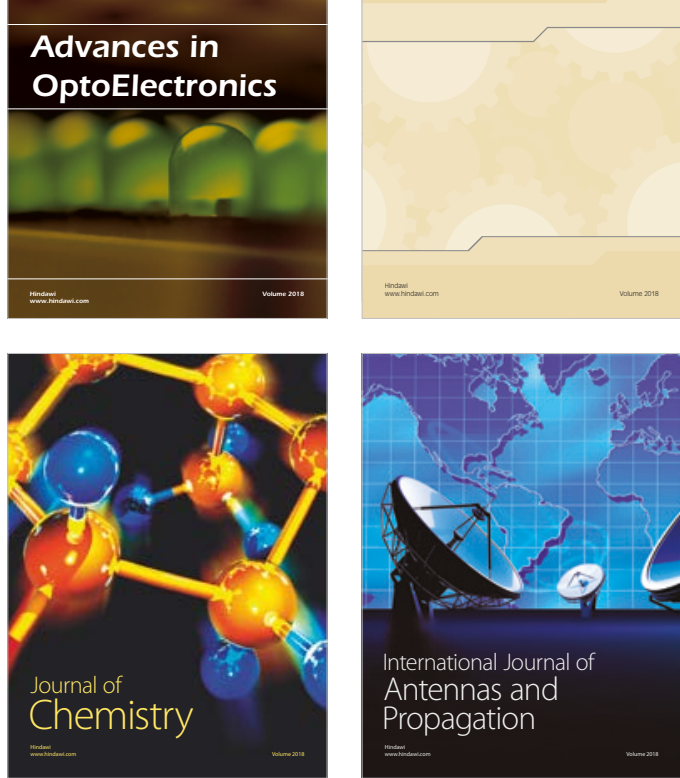

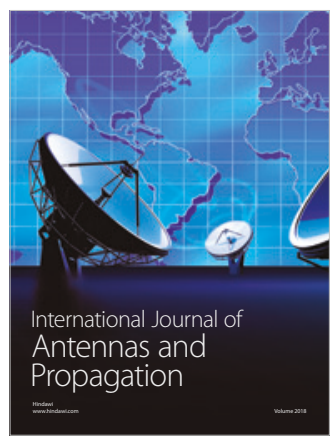

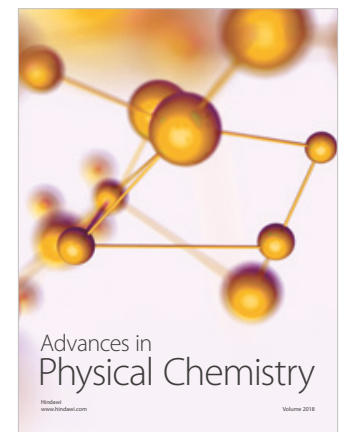

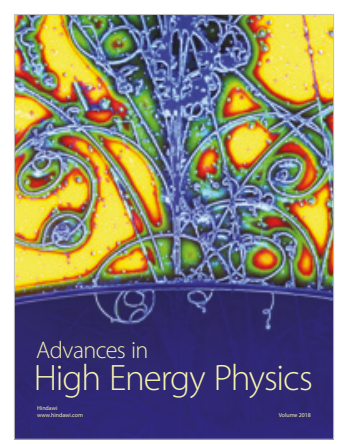

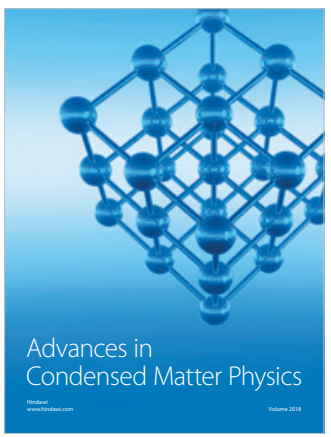

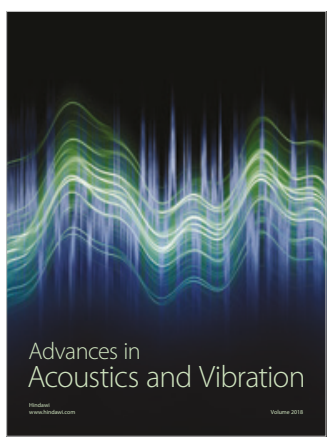

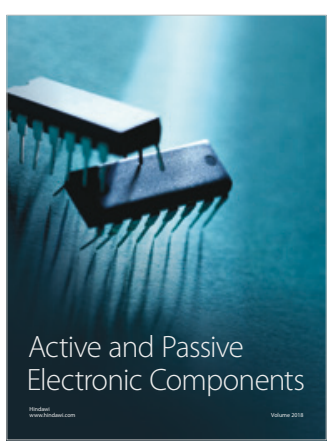
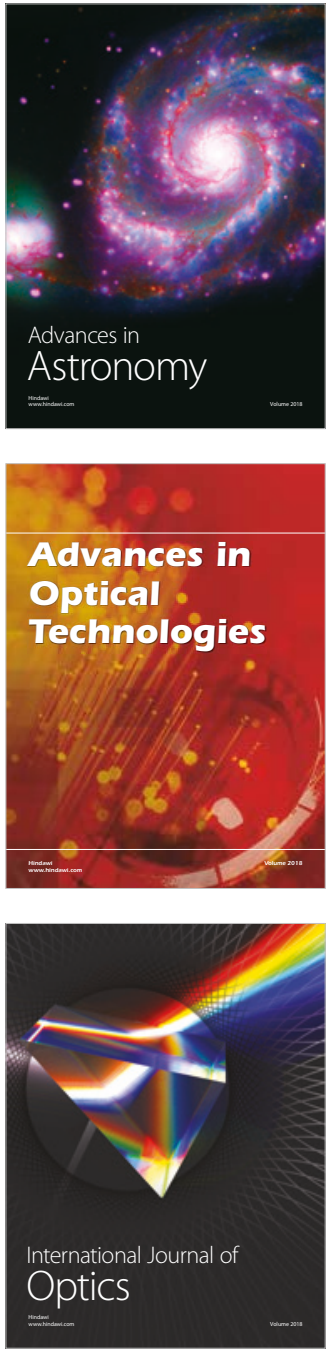